\title{
A millisecond pulsar candidate in a 21-hr orbit: 3FGL J0212.1+5320
}

\author{
Manuel Linares ${ }^{1,2,3 \star}$, Paulo Miles-Páez ${ }^{1,2}$, Pablo Rodríguez-Gil ${ }^{1,2}$, Tariq \\ Shahbaz $^{1,2}$, Jorge Casares ${ }^{1,2,4}$, Cecilia Fariña ${ }^{1,5}$, Raine Karjalainen ${ }^{5}$ \\ 1 Instituto de Astrofísica de Canarias, c/ Vía Láctea s/n, E-38205 La Laguna, Tenerife, Spain \\ 2 Universidad de La Laguna, Departamento de Astrofísica, E-38206 La Laguna, Tenerife, Spain \\ 3 Institutt for fysikk, NTNU, Trondheim, Norway \\ 4 Department of Physics, Astrophysics, University of Oxford, Denys Wilkinson Building, Keble Road, Oxford OX1 3RH, UK \\ 5 Isaac Newton Group of Telescopes, E-38700 S.C. de La Palma, Tenerife, Spain
}

24 November 2016

\begin{abstract}
We present the discovery of a variable optical counterpart to the unidentified gamma-ray source 3FGL J0212.1+5320 and argue this is a new compact binary millisecond pulsar (MSP) candidate. We show 3FGL J0212.1+5320 hosts a semi-detached binary with a $0.86955 \pm 0.00015 \mathrm{~d}$ orbital period and a F6-type companion star at an estimated distance of $\mathrm{D}=1.1 \pm 0.2 \mathrm{kpc}$, with a radial velocity curve semi-amplitude $\mathrm{K}_{2}=214.1 \pm 5.0 \mathrm{~km} \mathrm{~s}^{-1}$ and a projected rotational velocity of $\mathrm{V} \sin (\mathrm{i})=73.2 \pm 1.6 \mathrm{~km} \mathrm{~s}^{-1}$. We find a hard X-ray source at the same location with a $0.5-10 \mathrm{keV}$ luminosity $\mathrm{L}_{\mathrm{X}}=2.6 \times 10^{32}(\mathrm{D} / 1.1 \mathrm{kpc})^{2} \mathrm{erg} \mathrm{s}^{-1}$, which strengthens the MSP identification. Our results imply a mass ratio $\mathrm{q}=\mathrm{M}_{2} / \mathrm{M}_{1}=0.26_{-0.03}^{+0.02}$ if the companion star fills its Roche lobe, and $\mathrm{q} \gtrsim 0.26$ in any case. This classifies 3FGL J0212.1+5320 as a "redback" binary MSP; if its MSP nature is confirmed, this will be the brightest compact binary MSP in the optical band $\left(\mathrm{r}^{\prime} \simeq 14.3 \mathrm{mag}\right)$ and will have the longest orbital period among Galactic field systems (nearly $21 \mathrm{hr}$ ). Based on the light curve peak-to-peak amplitude $(\Delta \mathrm{r}=0.19 \mathrm{mag})$, we further suggest that the orbital inclination is high and the putative pulsar mass is close to canonical $\left(\mathrm{M}_{1} \simeq 1.3-1.6 \mathrm{M}_{\odot}\right)$. Finally, we discuss the lack of heating signatures and asymmetric optical light curves in the context of other redback MSPs.
\end{abstract}

Key words: stars: individual(3FGL J0212.1+5320) — gamma rays: stars — binaries: general — pulsars: general — stars: neutron — stars: variables: general

\section{INTRODUCTION}

Nearly one thousand gamma-ray sources from the Fermi Large Area Telescope (LAT) catalog remain unindentified, about a third of the total sample (Acero et al. 2015). This is often due to the lack of counterparts at longer wavelengths, and offers an appealing discovery space. Among the identified Galactic sources, pulsars are the most numerous class (Nolan et al. 2012; Abdo et al. 2013), and Fermi-LAT is uncovering a new population of nearby binary millisecond pulsars (MSPs; see, e.g., Hessels et al. 2011; Ray et al. 2012; Roberts 2013).

Dynamical studies of a few MSPs in compact binaries ("black-widow" and "redback" pulsars) have

* Linares@iac.es revealed evidence for massive neutron stars, with masses well above the canonical value of $1.4 \mathrm{M}_{\odot}$ van Kerkwiik, Breton \& Kulkarni 2011; Romani et al. 2012; Kaplan et al. 2013). These and related pulsar discoveries have pushed the maximum neutron star mass to more than two solar masses (Demorest et al. 2010; Antoniadis et al. 2013), placing tighter constraints on the equation of state above nuclear saturation density. Finding more such systems is crucial to establish their properties as a class, and constitutes a promising first step towards identifying the most massive neutron stars.

Radio timing observations of Fermi-LAT sources have unveiled a flurry of new pulsars (Hessels et al. 2011; Rav et al. 2012). However, black-widow and redback MSPs are often occulted for a large fraction of the orbit (Archibald et al. 2013), making their direct detection as ra- 

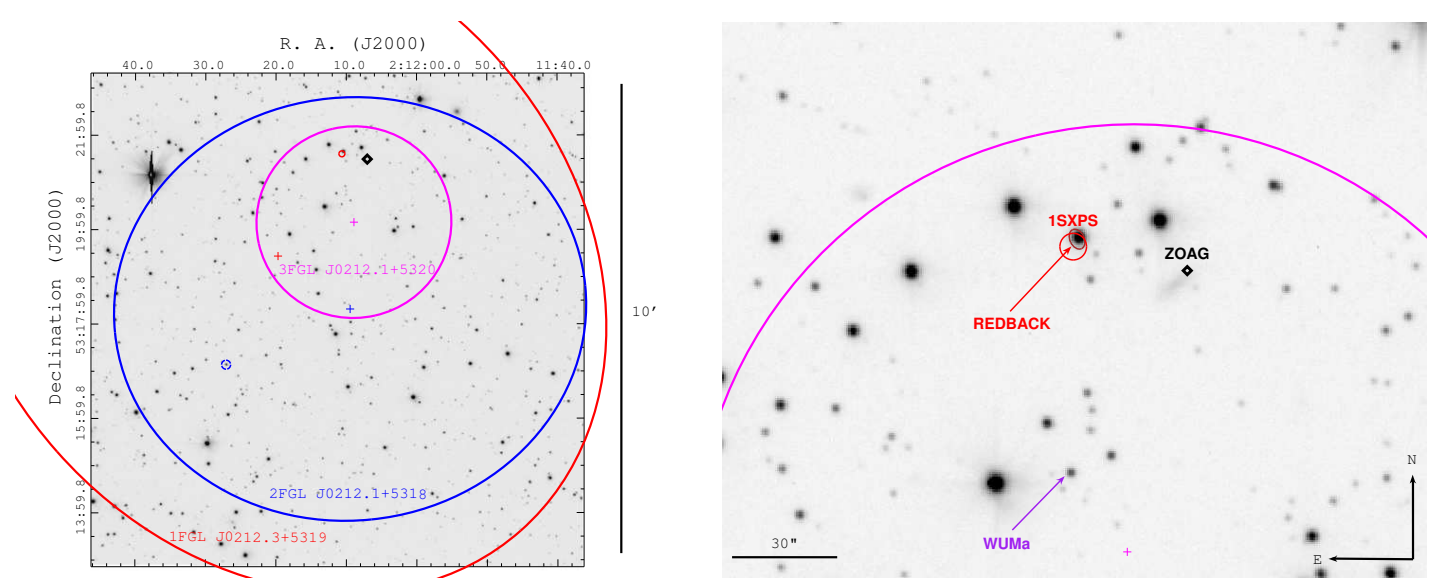

Figure 1. Left: Full IAC-80-CAMELOT r' band image of the field of 3FGL J0212.1+5320, showing the (95\%) error ellipses from each of the three Fermi-LAT point source catalogs, as indicated. Right: Zoomed finding chart showing the location of our variable optical source and redback candidate J0212 ( $\mathrm{P}_{\text {orb }} \simeq 20.9 \mathrm{hr}, \mathrm{r}^{\prime} \simeq 14.3 \mathrm{mag}$; red arrow), the Swift X-ray counterpart 1SXPS J021210.6+532136 (red circle), the nearby W UMa contact binary discovered in this work $\left(\mathrm{P}_{\text {orb }} \simeq 7.5 \mathrm{hr}, \mathrm{r}\right.$ ' $17 \mathrm{mag}$, purple arrow; see Appendix $\mathrm{A}$ ) and the nearby galaxy ZOAG G134.92-07.63 (black diamond). The brown ellipse shows the Chandra 3-sigma location.

dio pulsars challenging. Blind searches for gamma-ray pulsations have met with some success (Pletsch et al. 2012), yet they are computationally challenging, especially when the signal is smeared out by Doppler shifts in short (but unknown) orbital period binaries.

Here we take another approach to identify the FermiLAT source 3FGL J0212.1+5320, similar to that of Romani (2012) and Kong et al. (2012): we search for and find a variable optical counterpart (Section 2.1) that matches a previously unidentified X-ray source (Sec. 2.4). Our spectroscopic study (Section 2.2) allows us to measure the orbital period, the amplitude of the radial velocity curve, as well as the companion's spectral type and projected rotational velocity. Together with the multi-wavelength properties of the source, which we present in the rest of Section 2, this strongly suggests that the binary hosts a recycled "redback" MSP. We discuss the system's orbital parameters, potential and peculiarities in Section 3

\section{DATA ANALYSIS AND RESULTS}

\subsection{Optical Photometry}

We observed the field of 3FGL J0212.1+5320 with the CAMELOT camera mounted on the $82 \mathrm{~cm}$ IAC- 80 telescope, at the Teide Observatory. As shown in Fig. 11 the $10^{\prime} \times 10^{\prime}$ field of view covers all of the $2 \mathrm{FGL}$ and $3 \mathrm{FGL}$ location regions. We observed 3FGL J0212.1+5320 in three epochs, 2014 August, 2015 February and 2015 December, obtaining 1-3 minute-long exposures with the SDSS g'r'i' filters. On 2015 February 14 we also calibrated the field against two photometric standards, in photometric conditions. The resulting photometric data set is summarized in Table 1

\subsubsection{Variability search and source identification}

We used the 2014-08-02/03 and 2015-12-11 observations to search for variable counterparts, which had the longest un- interrupted sequences of r' images lasting about 2 and 7 hours, respectively. We identified 1296 objects in the field with signal-to-noise ratio $\geqslant 10$ and performed circular aperture photometry using the package PHOT-IRAF and an aperture radius equal to the average full width at half maximum (FWHM) of each image. We carefully selected nine stars that remained stable and used them as reference stars to perform differential photometry on the remaining objects in the field. We then measured the standard deviation $(\sigma)$ and the average value of the differential magnitude $(\Delta m)$ from the light curve of each object. We estimate that our search for variability is sensitive down to a r' magnitude of $\sim 20$, while our faintest detected sources had magnitudes of nearly 22 .

We found two strongly variable objects, with $\sigma \gtrsim 70 \mathrm{mmag}$, much more variable than the other objects at similar $\Delta m$ (which typically show $\sigma=[1-3] \mathrm{mmag}$ ). We identify the most variable star (with a peak-to-peak light curve amplitude $\Delta \mathrm{g}^{\prime} \simeq 0.4 \mathrm{mag}$ ) as a W UMa-type contact binary in the line of sight, as detailed in Appendix A This is shown with a purple arrow in Fig. 1 (right). We also found a much less variable object $\left(<14 \mathrm{mmag} \mathrm{hr}^{-1}\right)$ outside the error circle of 3FGL J0212.1+5320 (4.1' SE, about two times the $95 \%$ error radius), which we deem unrelated to the gammaray source. This is shown with a dashed small blue circle in Fig. 1 (left).

We find that the second most variable object, with $\Delta \mathrm{g}^{\prime} \simeq 0.2 \mathrm{mag}$, is coincident with an X-ray source (red circle and arrow and brown ellipse in Fig. 1). Based on its optical, X-ray and multi-wavelength properties, we argue that this is the counterpart to 3FGL J0212.1+5320 and a "redback" binary MSP. Using our astrometry-corrected 2015-1211 r'-band images, we locate the newly identified source at R.A. $=02^{h} 12^{m} 10.46^{s}, \mathrm{DEC}=+53^{\circ} 21^{\prime} 38.6^{\prime \prime}$ (J2000), with an $0.4^{\prime \prime}$ error radius (FWHM/2). Hereafter, we refer to this variable optical counterpart to 3FGL J0212.1+5320 as simply J0212. 


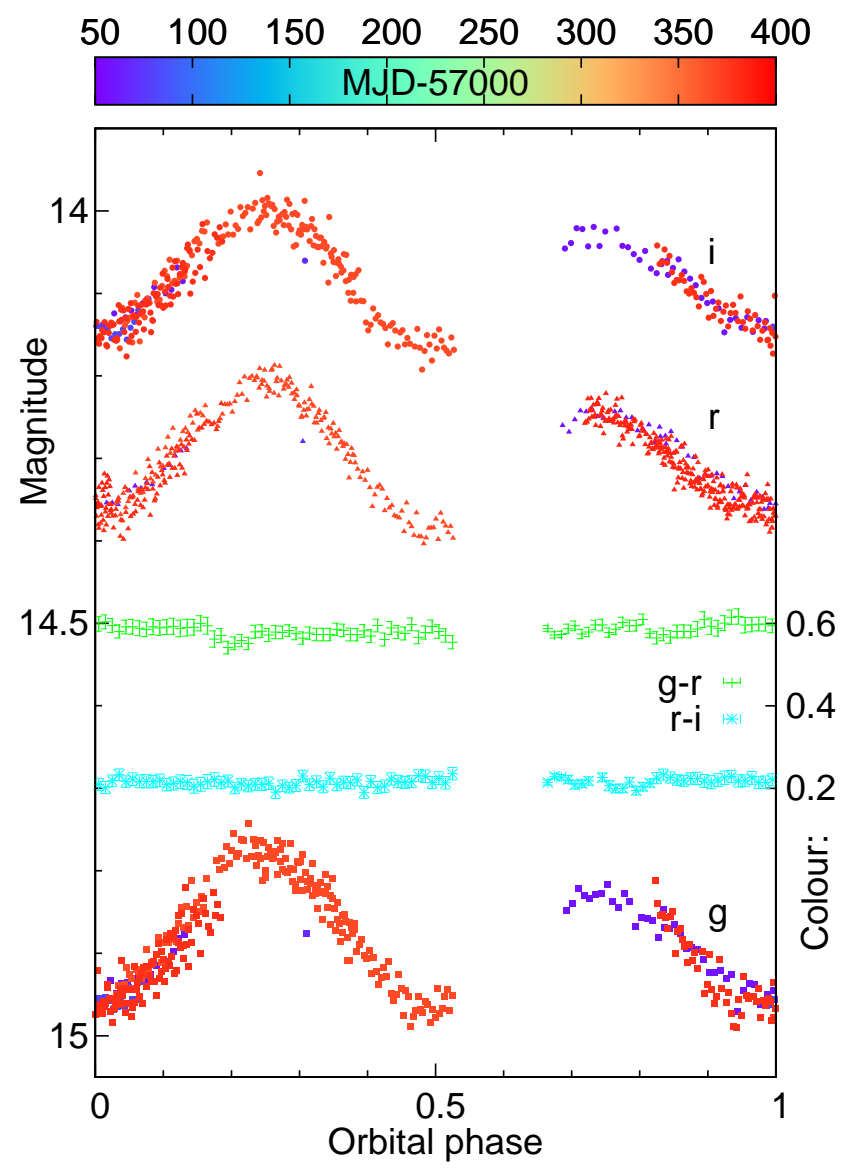

Figure 2. Optical g'r'i' light curves of J0212 from our two photometric epochs (February and December 2015; MJD date shown with colour scale, as indicated), folded at the orbital period $(0.86955 \mathrm{~d})$. The green and cyan symbols show the (g'-r') and $\left(\mathrm{r}^{\prime}-\mathrm{i}\right.$ ') colours, respectively, with the scale indicated along the right axis. The optical light curves are clearly asymmetric and show no significant colour changes.

\subsubsection{Light curves of the redback MSP candidate}

We performed differential photometry of our variable object J0212 and a nearby stable comparison star, using an aperture radius of 1.5-1.7 times the seeing. We calibrated the g'r'i' magnitudes of the comparison star with observations of the standard Hilt 233 (Smith et al. 2002) taken on the same photometric night (2015 February 14) and using the colour and extinction coefficients given by the IAC80-CAMELOT team ${ }^{1}$.

The light curves of J0212 in all three bands show qualitatively similar variability: smooth broad asymmetric maxima and minima (see Fig. 2). Besides this variability, indicative of orbital modulation in semi-detached compact binaries (e.g., Avni \& Bahcall 1975; Shahbaz et al. 1996; Orosz \& Bailyn 1997; Breton et al. 2013), J0212's light curves are stable on time-scales of days to months. We fitted the February-December 2015 light curves with a sine

1 http://www.iac.es/telescopes/pages/es/inicio/utilidades.php \#camelot-calibracion

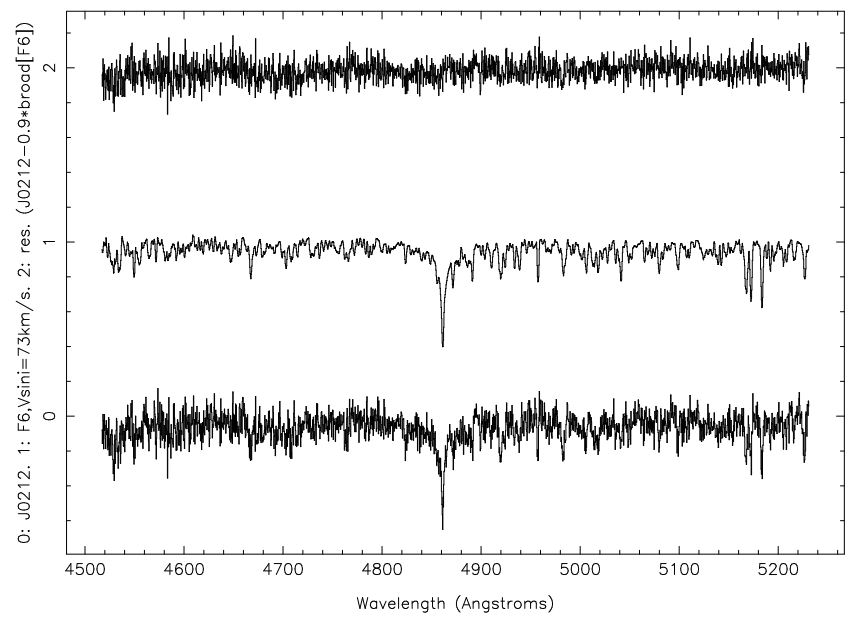

Figure 3. Average normalized INT-IDS-H1800V spectrum of J0212 shifted to the companion's reference frame (bottom), compared with an F6 template spectrum broadened by $\mathrm{V} \sin (\mathrm{i})=73 \mathrm{~km} \mathrm{~s}^{-1}$ (middle). Residuals from our best-match optimal subtraction are also shown (top).

function and obtained a photometric period $\mathrm{P}_{\mathrm{phot}} \simeq 0.4348 \mathrm{~d}$, consistent with $\mathrm{P}_{\text {orb }} / 2$ (see Section 2.2 below for details). The average magnitudes we find are 14.89, 14.30 and 14.08 in the g', r' an i' bands, respectively.

\subsection{Optical Spectroscopy}

We obtained 230 medium-to-low resolution spectra of J0212 with the William Herschel (WHT), Isaac Newton (INT) and Nordic Optical (NOT) telescopes at the Roque de los Muchachos observatory, on La Palma. The spectra were taken between December 2015 and March 2016, with exposure times, central resolutions and instrumental setups summarized in Table 1 We reduced these using standard IRAF routines, extracted them optimally with STARLINK/PAMELA (Marsh 1989) and calibrated the wavelength scale with interspersed arc lamp $(\mathrm{Ne} / \mathrm{Th} / \mathrm{Cu} / \mathrm{Ar})$ spectra, fitting a polynomial to the wavelength-pixel relation (giving residuals with rms more than 10 times smaller than the dispersion). We then fine-tuned the wavelength scale taking the $5577.338 \AA$ and $6300.304 \AA$ sky emission lines as reference, thereby correcting for sub-pixel offsets when present, and normalized the spectra dividing by a spline fit to the continuum.

\subsubsection{Spectral type and rotational velocity}

The spectra of J0212 feature hydrogen Balmer and metallic lines typical of F-type main-sequence stars. We applied the optimal subtraction method (Casares et al. 1993) in order to measure the spectral type and temperature of J0212, comparing quantitatively its photospheric absorption line spectra to a set of UVESpop main sequence templates covering spectral types O-M (Bagnulo et al. 2003). In all cases the spectra were shifted to the template rest frame to remove the orbital velocity of J0212 (see below). We find a spectral type of $\mathrm{F} 6 \pm 2$ from the 6 highest resolution spectra taken on 2015-12-23 (which cover orbital phases 0.17-0.22) using the hydrogen $\mathrm{H} \beta$ (4861 $\AA$ ), MgI triplet $(5167-5184 \AA)$ and 
Table 1. Summary of optical observations of 3FGL J0212.1+5320 (J0212).

\begin{tabular}{|c|c|c|c|c|c|c|c|}
\hline $\begin{array}{l}\text { Telescope } \\
\text { (diameter) }\end{array}$ & $\begin{array}{c}\text { Instrument } \\
\text { (configuration) }\end{array}$ & $\begin{array}{l}\operatorname{Band}^{a} \\
\text { (filt., } \bar{\AA} \text { ) }\end{array}$ & $\begin{array}{c}\text { Date } \\
\text { (evening) }\end{array}$ & $\begin{array}{l}\text { Time } \\
\text { (UT) }\end{array}$ & $\begin{array}{c}\text { Exposures } \\
(\mathrm{nr} . \times \text { duration })\end{array}$ & Airmass & $\begin{array}{l}\text { Seeing/Resolution } \\
\left(" / \mathrm{km} \mathrm{s}^{-1}\right)\end{array}$ \\
\hline \multicolumn{8}{|c|}{ Photometry } \\
\hline IAC $80-0.8 \mathrm{~m}$ & CAMELOT-2×2 & $\mathrm{r}^{\prime}$ & 2014-08-02 & 04:09-05:37 & $15 \times 300 \mathrm{~s}$ & $1.24-1.12$ & 1.1-1.7" \\
\hline IAC $80-0.8 \mathrm{~m}$ & CAMELOT- $2 \times 2$ & $r^{\prime}$ & 2014-08-03 & $04: 30-05: 20$ & $10 \times 300 \mathrm{~s}$ & $1.19-1.13$ & $0.7-0.8^{\prime \prime}$ \\
\hline IAC $80-0.8 \mathrm{~m}$ & CAMELOT-1x1 & g'r'i' & 2015-02-04 & $20: 03-23: 17$ & $54 \times 180$ s & $1.17-1.90$ & n.a. \\
\hline IAC $80-0.8 \mathrm{~m}$ & CAMELOT-1x1 & g'r'i' & 2015-02-05 & $19: 54-23: 13$ & $58 \times 180 \mathrm{~s}$ & $1.16-1.91$ & n.a. \\
\hline IAC $80-0.8 \mathrm{~m}$ & CAMELOT-1x1 & g'r'i' & 2015-02-06 & $19: 58-23: 08$ & $49 \times 180 \mathrm{~s}$ & $1.17-1.90$ & n.a. \\
\hline IAC $80-0.8 \mathrm{~m}$ & CAMELOT-1x1 & g'r'i' & 2015-02-14 & $22: 32-22: 39$ & $3 \times 180 \mathrm{~s}$ & $1.86-1.92$ & n.a. \\
\hline IAC $80-0.8 \mathrm{~m}$ & CAMELOT-1x1 & g'i' & 2015-02-26 & $20: 13-21: 47$ & $28 \times 180 \mathrm{~s}$ & $1.39-1.89$ & n.a. \\
\hline IAC $80-0.8 \mathrm{~m}$ & CAMELOT-1x1 & g'r'i' & 2015-12-10 & $19: 43-03: 04$ & $229 \times 60$ s & $1.10-1.96$ & n.a. \\
\hline IAC $80-0.8 \mathrm{~m}$ & CAMELOT-1x1 & g'r'i' & $2015-12-11$ & $19: 41-02: 57$ & $289 \times 60 \mathrm{~s}$ & $1.11-1.93$ & $0.6-1.6 "$ \\
\hline IAC $80-0.8 \mathrm{~m}$ & CAMELOT-1x1 & g'r'i' & $2015-12-22$ & 19:35-02:09 & $261 \times 60 \mathrm{~s}$ & $1.10-1.90$ & n.a. \\
\hline IAC $80-0.8 \mathrm{~m}$ & CAMELOT-1x1 & g'r'i' & $2015-12-23$ & 19:52-00:01 & $166 \times 60 \mathrm{~s}$ & $1.10-1.31$ & n.a. \\
\hline IAC $80-0.8 \mathrm{~m}$ & CAMELOT-1x1 & $\mathrm{r}^{\prime}$ & $2015-12-28$ & 19:30-01:51 & $257 \times 60$ s & $1.10-1.94$ & n.a. \\
\hline \multicolumn{8}{|c|}{ Spectroscopy } \\
\hline WHT $-4.2 \mathrm{~m}$ & ACAM-VPH400 & $4100-9000$ & 2015-12-16 & $19: 07-20: 49$ & $6 \times 120 \mathrm{~s}[2]^{b}$ & $1.12-1.26$ & 500 \\
\hline INT-2.5m & IDS-H1800V & $4400-5400$ & $2015-12-23$ & 23:59-01:01 & $6 \times 600 \mathrm{~s}$ & $1.29-1.46$ & 45 \\
\hline INT- $2.5 \mathrm{~m}$ & IDS-R400V & $4000-7700$ & 2016-01-19 & $20: 32-00: 22$ & $54 \times 400 \mathrm{~s}[2]$ & $1.13-1.64$ & 155 \\
\hline INT-2.5m & IDS-R400V & $4000-7100$ & 2016-01-20 & $20: 26-23: 59$ & $57 \times 200 \mathrm{~s}[2]$ & $1.12-1.71$ & 155 \\
\hline INT-2.5m & IDS-R400V & $4000-7100$ & 2016-01-21 & 19:45-00:01 & $28 \times 200 \mathrm{~s}[2]$ & $1.10-1.75$ & 155 \\
\hline INT-2.5m & IDS-R $400 \mathrm{~V}^{c}$ & $4000-7400$ & 2016-01-26 & $20: 46-23: 06$ & $18 \times 400 \mathrm{~s}[3]$ & $1.18-1.52$ & 155 \\
\hline NOT- $2.5 \mathrm{~m}$ & ALFOSC-G7-1" & $4000-6800$ & 2016-02-03 & $20: 39-00: 04$ & $24 \times 400 \mathrm{~s}$ & $1.21-2.19$ & 450 \\
\hline INT-2.5m & IDS-R632V & $4200-6700$ & 2016-02-25 & $20: 56-23: 10$ & $12 \times 550 \mathrm{~s}$ & $1.53-2.62$ & 140 \\
\hline NOT-2.5m & ALFOSC-G7-0.5" & $4000-6800$ & 2016-03-09 & $20: 31-21: 16$ & $6 \times 500 \mathrm{~s}$ & $1.63-1.94$ & 240 \\
\hline NOT-2.5m & ALFOSC-G7-0.5" & $4000-6800$ & 2016-03-14 & $21: 31-22: 16$ & $6 \times 500 \mathrm{~s}$ & $2.29-2.98$ & 240 \\
\hline NOT-2.5m & ALFOSC-G7-0.5" & $4000-6800$ & 2016-03-15 & $21: 56-22: 41$ & $6 \times 500 \mathrm{~s}$ & $2.69-3.68$ & 240 \\
\hline NOT-2.5m & ALFOSC-G7-0.5" & $4000-6800$ & 2016-03-16 & $21: 36-22: 22$ & $7 \times 500 \mathrm{~s}$ & $2.45-3.30$ & 240 \\
\hline
\end{tabular}

$a$ Photometric filters (effective wavelengths in $\AA$ : $\mathrm{g}^{\prime}=4639, \mathrm{r}^{\prime}=6122, \mathrm{i}^{\prime}=7439$ ) or wavelength range covered by the spectra, in $\AA$.

$b$ Very faint spectra were grouped by the numbers shown in brackets.

${ }^{c}$ Using the EEV instead of the RED+2 CCD used in all other IDS spectra.

other metallic absorption lines in the $4500-5300 \AA$ range (see Fig. 3). Lower resolution IDS spectra taken around orbital phases 0.5 and 0.75 also indicate a $\mathrm{F} 4-\mathrm{F} 8$ spectral type, or an effective temperature $\mathrm{T}_{\text {eff }}=6640-6150 \mathrm{~K}$. Thus we do not find evidence for temperature changes along the orbit larger than about $500 \mathrm{~K}$.

Applying the same method with a set of template spectra broadened by $[0-200] \mathrm{km} \mathrm{s}^{-1}$ in steps of $10 \mathrm{~km} \mathrm{~s}^{-1}$, we also measured the projected rotational velocity of the companion star in J0212, Vsin(i). Using F4, F6 and F8 templates we get consistent results for $\mathrm{V} \sin (\mathrm{i}): 71.8 \pm 3.1 \mathrm{~km} \mathrm{~s}^{-1}$, $73.6 \pm 2.7 \mathrm{~km} \mathrm{~s}^{-1}$ and $73.9 \pm 2.6 \mathrm{~km} \mathrm{~s}^{-1}$, respectively (where the best value was calculated from a parabolic fit to the lowest $3-5$ points and the errors correspond to $\Delta \chi^{2}=1$ ). We adopt the weighted average of these three values as our final measurement of $\mathrm{V} \sin (\mathrm{i})=73.2 \pm 1.6 \mathrm{~km} \mathrm{~s}^{-1}$. The best-match template spectra were multiplied by a factor $0.7-0.9$, which suggests a non-stellar light veiling of $10-30 \%$ in this band (approximately equivalent to the photometric SDSS filter g').

\subsubsection{Radial velocity curve}

The rest of the spectra were cross correlated with a set of $F$ templates in order to measure radial velocities along the orbit, keeping only cross-correlation values larger than 100 and adding a systematic error of $20 \mathrm{~km} \mathrm{~s}^{-1}$ to the statistical errors to account for residual uncertainties in absolute wavelength calibration. We included the $\mathrm{H} \alpha$ range (6513-
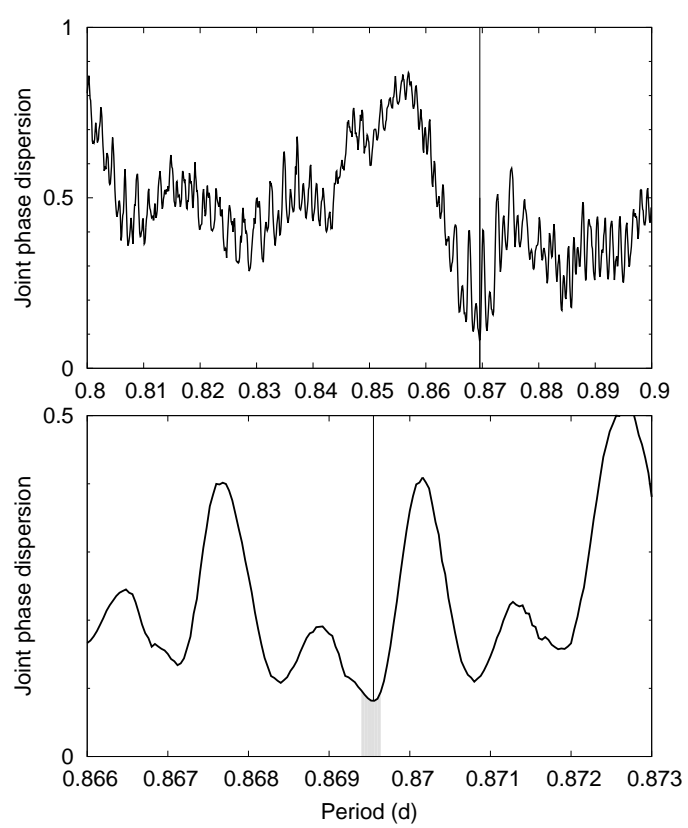

Figure 4. Top: Phase dispersion diagram of J0212, using all available observations (radial velocity and three-band light curves). Bottom: Zoom into the strongest minimum at the orbital period $\mathrm{P}_{\text {orb }}=0.86955 \pm 0.00015 \mathrm{~d}$, shown in gray and with the vertical line (Sec. 2.2.2). 


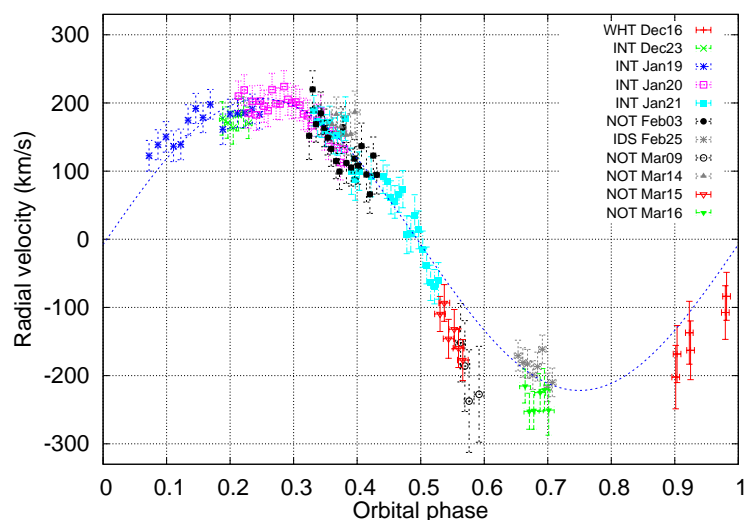

Figure 5. Radial velocity curve of J0212, from cross-correlating the December 2015- March 2016 spectra of J0212 with an F6 template (Sec. 2.2 for details). The best-fit sinusoidal function with $\mathrm{K}_{2}=214.1 \pm 5.0 \mathrm{~km} \mathrm{~s}^{-1}$, T0 $=57408.539 \pm 0.003 \mathrm{MJD}(\mathrm{TDB})$, and $\gamma=-7.7 \pm 4.8 \mathrm{~km} \mathrm{~s}^{-1}$ is also shown.

$6613 \AA$ ) to calculate radial velocities consistently (except in the 2015-12-23 spectra where only the $\mathrm{H} \beta$ range was available), binned each series of spectra to match the corresponding dispersion and broadened the templates in each case to match the spectral resolution (Table 1).

After applying the barycentric correction to both photometric and spectroscopic data sets (Eastman, Siverd \& Gaudi 2010), we used the final set of 131 radial velocities and $629 \mathrm{r}$ ' band magnitudes in order to find and measure the orbital period. Due to nightly sampling and large data gaps, periodograms of the radial velocity curve (RVC) and light curve (LC) are severly affected by aliasing. Using phase-dispersion minimization (PDM; Stellingwerf 1978) techniques, we find the strongest periodicity in the RVC at $0.8693 \pm 0.0004 \mathrm{~d}$ (errors quoted use a $20 \%$ increase in PDM statistics). Although the RVC PDM diagram shows six other possible periods in the 0.8-0.9 d range, none of them produces a smooth folded $\mathrm{RVC}$, and we can rule them out. To refine this period, we use the barycentred g', r' and i' band LCs, which extend from February to December 2015. The combined LC+RVC PDM diagram (see Fig. 4) shows the strongest minimum at $\mathrm{P}_{\text {orb }}=0.86955 \pm 0.00015 \mathrm{~d}$, consistent with and more precise than the period obtained from the RVC only.

From a sine fit to the RVCs (Fig. 5), we measure a semi-amplitude of $\mathrm{K}_{2}=214.1 \pm 5.0 \mathrm{~km} \mathrm{~s}^{-1}$, a barycentric time of zero phase (companion at inferior conjunction) $\mathrm{T} 0=57408.539 \pm 0.003 \mathrm{MJD}(\mathrm{TDB})$, and a systemic velocity $\gamma=-7.7 \pm 4.8 \mathrm{~km} \mathrm{~s}^{-1}$. The errors reported here include the effect of using different templates for the cross-correlation analysis, with spectral types F4-F8.

\subsection{Gamma-ray}

As can be seen in Fig. 11 the location of the 0.1-100 GeV gamma-ray source has improved with the increased exposure, when going from the first (1FGL J0212.3+5319) to the second (2FGL J0212.1+5318) and third (3FGL J0212.1+5320) Fermi-LAT catalogues (Abdo et al. 2010; Nolan et al. 2012; Acero et al. 2015). The LAT location converges towards the position of J0212, our variable optical counterpart (Sec. 2.1). Unless otherwise noted, we give in this section the most updated values from the third Fermi-LAT source catalog. The LAT error region is nearly circular with a $2^{\prime}$ radius, and the spectrum is clearly curved (log-normal in the description of Acero et al. 2015, with significant curvature, 6.3 , and a pivot energy of $\sim 1.1 \mathrm{GeV}$; see Fig. 6).

The LAT flux is relatively stable on timescales of weeksmonths, with a variability index of 51.5 (17.3 in the second catalog). These characteristics (curved spectrum and low variability) are similar to the known Fermi-LAT pulsar population (e.g., Abdo et al. 2013), which is why we selected 3FGL J0212.1+5320 for the present work. The integrated $0.1-100 \mathrm{GeV}$ energy flux is $[1.71 \pm 0.16] \times 10^{-11} \mathrm{erg} \mathrm{cm}^{-2} \mathrm{~s}^{-1}$ Acero et al. 2015). The gamma-to-X-ray flux ratio $(\sim 10)$ is consistent with other redback MSPs. For the distance (D) derived in Section 3 the corresponding gamma-ray (0.1$100 \mathrm{GeV})$ luminosity $\mathrm{L}_{\gamma}=2.5 \times 10^{33}(\mathrm{D} / 1.1 \mathrm{kpc})^{2} \mathrm{erg} \mathrm{s}^{-1}$ is also typical of MSPs (most of which have $\mathrm{L}_{\gamma} \sim 10^{32}$ $10^{34} \mathrm{erg} \mathrm{s}^{-1}$; Abdo et al. 2013).

\subsection{X-ray}

Chandra (ACIS-S) observed the field on $2013 \mathrm{Au}-$ gust 22 for a total of $30 \mathrm{ks}$. We find an X-ray point source at R.A. $=02^{h} 12^{m} 10.50^{s}, \mathrm{DEC}=+53^{\circ} 21^{\prime} 38.9^{\prime \prime}$ (J2000), with an estimated $0.6^{\prime \prime}$ uncertainty 2. As shown in Fig. 1 (brown ellipse), this is in excellent agreement with the position of our variable optical counterpart, J0212. The background-corrected $0.5-10 \mathrm{keV}$ spectrum is well fit $\left(\chi^{2} /\right.$ d.o.f. $\left.=134.3 / 142\right)$ with an absorbed power law model (Fig. 6). We measure a photon index $\Gamma=1.29 \pm 0.06$, an absorbing equivalent hydrogen column $\mathrm{N}_{\mathrm{H}}=[1.4 \pm 0.3] \times 10^{21} \mathrm{~cm}^{-2}$ and an unabsorbed $0.5-10 \mathrm{keV}$ flux of $[1.8 \pm 0.1] \times 10^{-12} \mathrm{erg} \mathrm{s}^{-1} \mathrm{~cm}^{-2}$. The backgroundcorrected $0.3-10 \mathrm{keV}$ count rate stayed roughly constant at $\sim 0.1 \mathrm{c} \mathrm{s}^{-1}$ during the observation, which covered about $40 \%$ of an orbital cycle. J0212 was also detected during SwiftXRT observations on 2010 October 9 and 12 for a total exposure of $4.5 \mathrm{ks}$, at a position and X-ray flux consistent with the values reported above.

Taking as a reference the distance estimated in Section 3 the $0.5-10 \mathrm{keV}$ X-ray luminosity of $\mathrm{J} 0212$ is $\mathrm{L}_{\mathrm{X}}=2.6 \times 10^{32}(\mathrm{D} / 1.1 \mathrm{kpc})^{2} \mathrm{erg} \mathrm{s}^{-1}$. This $\mathrm{L}_{\mathrm{X}}$ and the very hard spectrum (photon index of 1.3) are fully consistent with the rest of nearby redbacks in the pulsar state Linares 2014). Orbital X-ray variability from J0212 may be observed with longer exposures, like that seen in other redbacks and interpreted as beaming or partial occultation of the intrabinary shock (e.g., Bogdanov et al. 2011).

\subsection{Ultraviolet}

Swift-UVOT observed the field in two occasions, on 2010 October 9 and 12, taking a 767s UV-M2 and a 1217s UVW2 exposure, respectively. We find a clear UV counterpart to J0212 in both filters; using a $5^{\prime \prime}$ aperture radius and

2 While our work was being completed, Saz Parkinson et al. 2016 tentatively identified this source, CXOU J021210.5+532138, as a probable counterpart to 3FGL J0212.1+5320. 


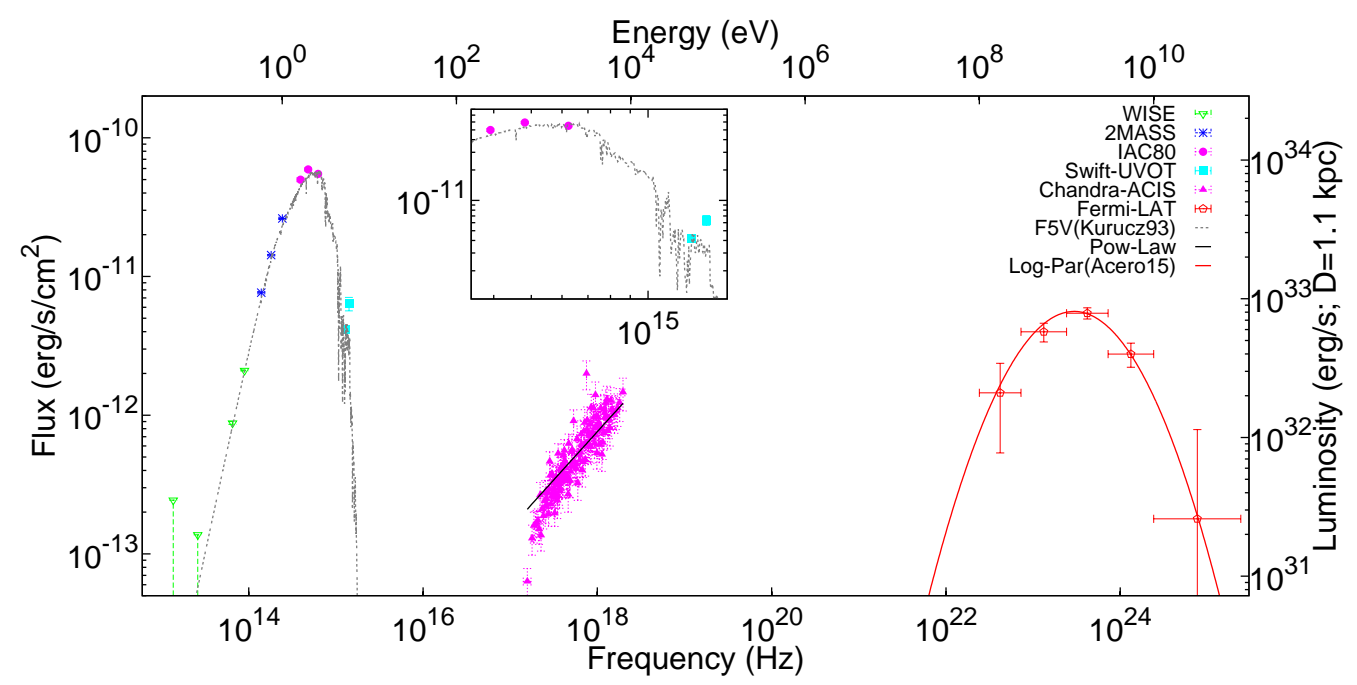

Figure 6. Broadband spectral energy distribution of J0212, from IR to gamma-rays. The optical fluxes correspond to phase 0 (Sec. 3), and the X-ray data show the Chandra spectrum (Sec. 2.4). Fluxes from IR to UV are corrected for interstellar absorption. For comparison, we show the spectrum of an F5V star with $1.2 \mathrm{R}_{\odot}$ radius at $\mathrm{D}=1.1 \mathrm{kpc}$ (dashed gray line, not fitted; from Kurucz 1993). We also plot the best-fit power-law model (black line, corrected for absorption; Sec. 2.4) and the log-parabola fit to the LAT spectrum (red line; from Acero et al. 2015). See Sections 2.12.6 for details and references.

the UVOT task UVOTSOURCE, we measure magnitudes of $17.99 \pm 0.05$ and $18.16 \pm 0.09$ in the W2 and M2 filters, respectively. The corresponding fluxes are shown in Fig. 6] after correcting for absorption (Sec. 33).

\subsection{Infrared}

Our variable optical counterpart matches a 2MASS source (2MASS J02121047+5321387; Skrutskie et al. 2006) with the following magnitudes: $\mathrm{J}=13.144 \pm 0.023$, $\mathrm{H}=12.915 \pm 0.020$ and $\mathrm{K}=12.797 \pm 0.019$. We also find a WISE source at the same location (WISE J021210.46+532138.7; Wright et al. 2010), with the following magnitudes: $w 1=12.807 \pm 0.025$, $\mathrm{w} 2=12.759 \pm 0.026$, w3 $>11.94$ and $\mathrm{w} 4>9.16$. The corresponding fluxes are shown in Fig. 6 ]

\section{DISCUSSION}

\subsection{Masses and orbital parameters}

We have discovered a variable optical counterpart to the gamma-ray source 3FGL J0212.1+5320, J0212, which coincides with a previously unclassified X-ray source. The multiwavelength properties of J0212 are consistent with a binary millisecond pulsar in a compact orbit $\left(\mathrm{P}_{\text {orb }}=20.869[4] \mathrm{h}\right)$ with a F6 \pm 2 main sequence companion star. From the measured $\mathrm{V} \sin (\mathrm{i})=73.2 \pm 1.6 \mathrm{~km} \mathrm{~s}^{-1}$ and $\mathrm{K}_{2}=214.1 \pm 5.0 \mathrm{~km} \mathrm{~s}^{-1}$ (Sec. 2.2), assuming a Roche-lobe filling, tidally locked and spherically symmetric companion star, we find a mass ratio $\mathrm{q}=\mathrm{M}_{2} / \mathrm{M}_{1}=0.26_{-0.03}^{+0.02}$ (where $\mathrm{M}_{2}$ and $\mathrm{M}_{1}$ are the masses of the secondary/companion and the primary/neutron star; see Wade \& Horne 1988). We note this is strictly a lower limit and thus $\mathrm{q} \gtrsim 0.26$, as the companion may be smaller than its Roche lobe.

These orbital parameters classify 3FGL J0212.1+5320

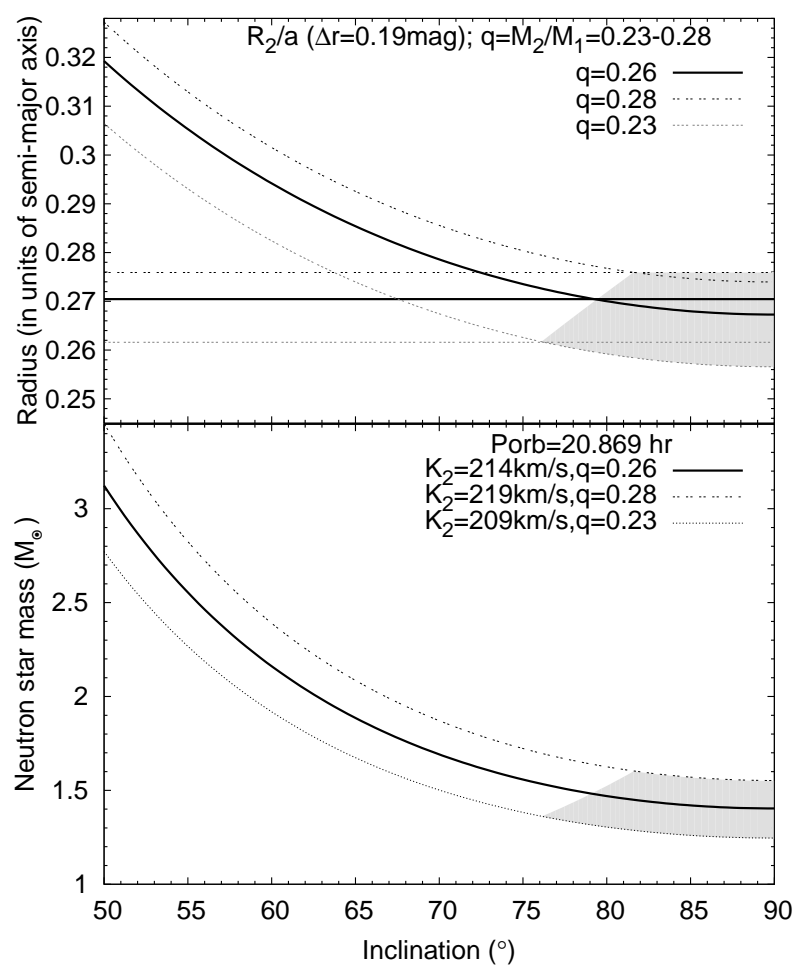

Figure 7. Bottom: Mass of the primary or neutron star as a function of inclination $\left(\mathrm{i}=90^{\circ}\right.$ corresponds to the orbital plane viewed edge on). The curves shown use the indicated values of $\mathrm{P}_{\mathrm{orb}}, \mathrm{K}_{2}$ and $\mathrm{q}$ (Sections 2.2 and 3.1). Top: Radius of the secondary or companion star as a function of inclination, in units of the semi-major axis a. Horizontal lines show the Roche lobe radius $R_{L 2}$ for our estimated values of $q$, as indicated. Curves show $R_{2} /$ a for the observed light curve amplitude $\Delta r=0.19$ mag (Morris 1985). Gray-shaded regions show the preferred ranges of $\mathrm{i}, \mathrm{M}_{1}$ and $\mathrm{R}_{2} / \mathrm{a}$ from imposing that $\mathrm{R}_{2} \leqslant \mathrm{R}_{\mathrm{L} 2}$ (Sec. 3.1). 
as a "redback" MSP (which have $\mathrm{M}_{2} \gtrsim 0.1-0.5 \mathrm{M}_{\odot}$; e.g., Roberts 2011). The measured absorbing column density (Sec. 2.4) corresponds to a pulsar dispersion measure $\mathrm{DM} \sim 50 \mathrm{pc} \mathrm{cm}{ }^{-3}$ according to the correlation presented in He, Ng \& Kaspi (2013). This optical ephemeris will allow targeted searches for radio (preferentially around phase 0.5 to avoid pulsar occultation) and gamma-ray millisecond pulsations from this system, not reported to date. 3FGL J0212.1+5320 does not appear in the NRT radio pulsar search of Fermi-LAT sources presented by Guillemot et al. (2012), and we find no radio counterpart in the NVSS $1.4 \mathrm{GHz}$ survey (Condon et al. 1998, the field was not covered by FIRST).

If its redback nature is confirmed, J0212 will have the longest $\mathrm{P}_{\text {orb }}$ among the compact binary millisecond pulsars in the Galactic field (both redbacks and black widows). To our knowledge, only two redbacks with longer orbital period are known, both residing in globular clusters: J1748-2446AD $\left(\mathrm{P}_{\text {orb }}=1.09 \mathrm{~d}\right)$ in Terzan 5 and J1740-5340 $\left(\mathrm{P}_{\text {orb }}=1.35 \mathrm{~d}\right)$ in NGC 6397 (Hessels et al. 2006; D'Amico et al. 2001, respectively). From our $K_{2}$ and q measurements we derive the $M_{1}-i$ relation shown in Fig. 7 (bottom; using $\mathrm{M}_{1}=(1+\mathrm{q})^{2} \mathrm{P} \mathrm{K}_{2}{ }^{3}$ / $\left.\left(2 \pi \mathrm{G} \sin ^{3}(\mathrm{i})\right)\right)$. If the primary is indeed a neutron star, our results imply that $\mathrm{i}>50^{\circ}$ and $\mathrm{M}_{2}<0.8 \mathrm{M}_{\odot}$ for any plausible $\mathrm{M}_{1}<3 \mathrm{M}_{\odot}$, and $\mathrm{M}_{1} \gtrsim 1.3 \mathrm{M}_{\odot}$ for any i.

Furthermore, since we find that irradiation effects are negligible in J0212 (Secs. 2.2 and 3.2), we can constrain the inclination by ascribing the observed light curve amplitude (peak-to-peak amplitude in r' $\Delta \mathrm{r}=0.19 \mathrm{mag}$ ) to ellipsoidal modulation of the tidally locked companion. Using the analytical method presented by Morris (1985, equation (6) in particular) and taking limb- and gravity-darkening coefficients for a Solar metallicity F5 star with $\log (\mathrm{g})=4.5$ Claret \& Bloemen 2011), we can constrain the companion radius $\mathrm{R}_{2}$ /a (Fig. 17, top), where a is the semi-major axis of the orbit. Imposing that $\mathrm{R}_{2}$ is smaller than the corresponding Roche lobe radius $\left(\mathrm{R}_{\mathrm{L} 2}\right.$; Eggleton 1983), since there is no evidence for mass transfer and accretion disk lines are not observed (Sec. 2.2), we find that $\mathrm{i} \gtrsim 76^{\circ}$ and therefore $\mathrm{M}_{1} \simeq[1.3-1.6] \quad \mathrm{M}_{\odot}$ and $\mathrm{M}_{2} \simeq[0.34-0.42] \quad \mathrm{M}_{\odot}$. Thus according to the Morris (1985) relation, the inclination should be high $\left(i \gtrsim 76^{\circ}\right)$ and the companion should be close to filling its Roche lobe $\left(R_{2} / R_{L 2}>98 \%\right)$ in order to produce the observed ellipsoidal modulation.

The $\mathrm{M}_{2}$ constraints above imply that the companion is significantly larger and hotter than an isolated star of its mass. If we take $\mathrm{M}_{1}=1.5 \mathrm{M}_{\odot}$ and $\mathrm{M}_{2}=0.38 \mathrm{M}_{\odot}$ $\left(\mathrm{i}=80^{\circ}\right.$ ), the corresponding $\mathrm{R}_{2} \simeq \mathrm{R}_{\mathrm{L} 2}=1.3 \mathrm{R}_{\odot}$ is roughly consistent with the radius of an isolated F6V star. Similar "stripped" or "bloated" companion stars, hotter and/or larger than isolated stars of the same mass, are seen in redback (Crawford et al. 2013) and black-widow MSPs (van Kerkwiik. Breton \& Kulkarni 2011), as well as neutron star transients in quiescence (Bildsten \& Chakrabartv 2001; see also Orosz \& van Kerkwiik 2003 for further discussion). Finally, we note that if the pulsar is detected, J0212 will be an ideal system for an accurate neutron star mass measurement: it has a bright, non-irradiated companion star in a likely high inclination orbit. Pulsar timing and highresolution spectroscopy can yield much more precise measurements of $\mathrm{q}$ and $\mathrm{K}_{2}$, respectively. Detailed modelling of the optical light curve and spectral lines can give tighter and more robust constraints on the inclination angle.

\subsection{Colours and broadband SED}

After correcting for interstellar reddening using $\mathrm{E}(\mathrm{B}-\mathrm{V})=$ $\mathrm{N}_{\mathrm{H}} /\left(3.1 \times 1.8 \times 10^{21} \mathrm{~cm}^{-2}\right)=0.251$ (with the $\mathrm{N}_{\mathrm{H}}$ measured from the X-ray spectrum; Sec. 2.4 and Predehl \& Schmitt 1995), the corresponding (g'-r') and (r'-i') colours are fully consistent with the F6 spectral type we find from optical spectroscopy (Pecaut \& Mamaiek 2013, see Sec. 2.2). The infrared, optical and UV fluxes are also consistent with an F6 main sequence star with radius $\simeq 1.3 \mathrm{R}_{\odot}$ at $\mathrm{D} \simeq 1.1 \mathrm{kpc}$, as shown in Fig. 6. We note that the W2 flux (the shortest wavelength UV measurement available, at $2120 \AA$ ) is about $40 \%$ higher than the M2 flux (at $2310 \AA$ ). Comparing with stellar atmosphere models of a F5V star (Fig. 6] Kurucz 1993), we attribute this to a relative drop in M2 flux due to FeII absorption bands in the $\sim 2300-2400 \AA$ range. The spectral energy distribution (Fig. 6) also shows the energy budget of the companion star $\left(\sim 10^{34} \mathrm{erg} \mathrm{s}^{-1}\right)$ which dominates in the optical band, the gamma-rays from the putative MSP $\left(\sim 10^{33} \mathrm{erg} \mathrm{s}^{-1}\right)$ and the shock between the MSP and companion winds (intrabinary shock), which presumably powers the X-ray emission $\left(\sim 10^{32} \mathrm{erg} \mathrm{s}^{-1}\right)$.

The colours remain approximately constant along the orbit (Fig. 2), implying little or no temperature change between the different sides of the companion. This complete lack of irradiation or "heating" of the companion by the pulsar wind and radiation is exceptional among compact binary MSPs. From the allowed range of temperatures (6640-6150 K; Sec. 2.2), a semi-major axis of $4.7 \mathrm{R}_{\odot}$ $\left(\mathrm{i}=80^{\circ}\right)$ and assuming that the pulsar spin-down power $\dot{E}$ is emitted isotropically, we estimate an upper limit on $\dot{E}<[1-$ $4] \times 10^{35} \mathrm{erg} \mathrm{s}^{-1}$ (for an irradiation efficiency 10-30\%, following Breton et al. 2013). This limit is consistent with the $\dot{E}$ of most MSPs. Thus we suggest that the lack of heating is simply due to the wide orbit of J0212. To our knowledge only PSR J1740-5340, which is also in a long $\mathrm{P}_{\text {orb }} \simeq 32 \mathrm{hr}$ orbit, has shown a similar lack of irradiation (Orosz \& van Kerkwijk 2003).

\subsection{Light curves and distance}

We measure phase-zero magnitudes of $14.96,14.36$ and 14.15 in the g', r' an i' bands, respectively, which correspond to a dereddened $\mathrm{V}=13.83$. This makes J0212 the brightest compact binary MSP known to date (about two magnitudes brighter than PSR J1723-2837 in V, Crawford et al. 2013). For an F6 main sequence star with absolute magnitude $\mathrm{M}_{\mathrm{V}}=3.7$ (Pecaut \& Mamajek 2013), implicitly assuming that the companion radius is unperturbed, we estimate a distance to J0212 of $\mathrm{D}=1.1 \pm 0.2 \mathrm{kpc}$ (where the error corresponds to the allowed range of spectral types, F4-F8). As discussed in Section 3.1. the radius we infer from our RVC and LC analysis is consistent with this spectral type (but the mass is not).

The optical orbital light curves are clearly asymmetric (Fig. 2): the light maximum at phase 0.25 (companion at ascending node) is $0.03,0.04$ and 0.06 magnitudes brighter than the maximum at phase 0.75 (descending node) 
in the $\mathrm{i}, \mathrm{r}$ and $\mathrm{g}$ bands, respectively. The minimum at phase 0 (companion at inferior conjunction) is about $0.01-$ 0.03 magnitudes brighter than the minimum at phase 0.5 . While the asymmetry in the depth of the minima might be partly explained by limb- and gravity-darkening effects, models for compact binary MSP light curves in general, and their asymmetric maxima in particular, are still under development (Breton et al. 2013; Li, Halpern \& Thorstensen 2014; Salvetti et al. 2015). We leave detailed modelling for future work, and simply point out that optical light curves similar to those of J0212 have been observed in confirmed and candidate redback MSPs (PSR J1628-32, $\mathrm{P}_{\text {orb }} \simeq 5 \mathrm{hr}$, Li, Halpern \& Thorstensen 2014; PSR J21290429, $\mathrm{P}_{\text {orb }} \simeq 15.2 \mathrm{hr}$, Bellm et al. 2016; 1FGL J0523.5-2529, $\mathrm{P}_{\text {orb }} \simeq 16.5 \mathrm{hr}$ ?, Strader et al. 2014; 3FGL J2039.6-5618, $\mathrm{P}_{\text {orb }} \simeq 5.4 \mathrm{hr}$ ?, Salvetti et al. 2015).

\subsection{X-rays and intrabinary shock}

J0212 features the highest X-ray luminosity $\left(2.6 \times 10^{32}\right.$ erg $\mathrm{s}^{-1}$; Sec. 2.4) among redback (in the pulsar state) and black-widow MSPs (Linares 2014). This places J0212 in the group of relatively X-ray luminous redbacks. PSR 1740-5340, on the other hand, had $\sim 10$ times lower $\mathrm{Lx}_{\mathrm{X}}$ (Bogdanov et al. 2010, and references therein). The wide orbit and lack of irradiation signatures strongly suggests that the companion wind in these systems is not driven by MSP heating effects. Thus while irradiation of the companion appears to depend critically on $\mathrm{P}_{\text {orb }}$, the luminosity of the intrabinary shock between the pulsar and companion winds is not simply related to $\mathrm{P}_{\text {orb }}$. This may be due to a hotter companion with a larger mass loss rate in the wind, which would compensate the larger orbital separation.

\subsection{Towards a systematic search}

To conclude, our results highlight the potential of smallaperture optical telescopes like the IAC80 in identifying and characterizing Fermi-LAT sources. The exceptionally bright optical counterpart to 3FGL J0212.1+5320 that we have discovered, with $\mathrm{r}^{\prime} \simeq 14.3 \mathrm{mag}$, sets the record for the brightest MSP with a low-mass companion. A complete photometric survey of unidentified Fermi-LAT sources should thus target a broad range spanning more than 12 magnitudes, from the faintest g' 27 mag black-widow counterparts (e.g., Breton et al. 2013) to the g' 15 mag of J0212 or brighter. It should be noted, moreover, that intra-night optical variability searches are biased towards short- $\mathrm{P}_{\text {orb }}$ and strongly irradiated systems, which give rise to drastic magnitude changes in only a few hours (e.g., Schroeder \& Halpern 2014). Our findings show that a systematic search for compact binary MSPs should also target low amplitude $\left(\lesssim 0.05 \mathrm{mag} \mathrm{hr}^{-1}\right)$ and long period ( $\gtrsim 12 \mathrm{hr}$ ) optical variability.

Note: Soon after our manuscript was submitted, Li et al. (arXiv:1609.02951) published a similar analysis of 3FGL J0212.1+5320. Our results mostly agree.

Acknowledgments: This article is based on observations made with the IAC80, WHT, INT and NOT telescopes operated on the islands of Tenerife and La Palma by the IAC, ING and NOTSA. We thank R. Alonso, R. Ashley,
M. Balcells, P. Blay, P. Chinchilla, E. Gómez, A. Oscoz, C. Protasio and J. Telting for granting, assisting or performing some of the observations presented here. IRAF is distributed by the NOAO, operated by AURA under cooperative agreement with the NSF. We gratefully acknowledge the use of T. Marsh's MOLLY, PAMELA and ULTRACAM analysis packages. This research was supported by the Spanish MINECO under grants AYA2013-42627 and AYA2012-38700. PRG is supported by a Ramón y Cajal fellowship (RYC2010-05762). JC acknowledges support by the Leverhulme Trust through the Visiting Professorship Grant VP2-2015-046. This publication makes use of data products provided by HEASARC, 2MASS and WISE.

\section{REFERENCES}

Abdo A. A. et al., 2010, ApJS, 188, 405

Abdo A. A. et al., 2013, ApJS, 208, 17

Acero et al. F., 2015, ApJS, 218, 23

Antoniadis J. et al., 2013, Science, 340, 448

Archibald A. M., Kaspi V. M., Hessels J. W. T., Stappers B., Janssen G., Lyne A., 2013, Submitted to ApJ; ArXiv 1311.5161

Avni Y., Bahcall J. N., 1975, ApJ, 197, 675

Bagnulo S., Jehin E., Ledoux C., Cabanac R., Melo C., Gilmozzi R., ESO Paranal Science Operations Team, 2003, The Messenger, 114, 10

Bellm E. C. et al., 2016, ApJ, 816, 74

Bildsten L., Chakrabarty D., 2001, ApJ, 557, 292

Bogdanov S., Archibald A. M., Hessels J. W. T., Kaspi V. M., Lorimer D., McLaughlin M. A., Ransom S. M., Stairs I. H., 2011, ApJ, 742, 97

Bogdanov S., van den Berg M., Heinke C. O., Cohn H. N., Lugger P. M., Grindlay J. E., 2010, ApJ, 709, 241

Breton R. P. et al., 2013, ApJ, 769, 108

Casares J., Charles P. A., Naylor T., Pavlenko E. P., 1993, MNRAS, 265, 834

Claret A., Bloemen S., 2011, A\&A, 529, A75

Condon J. J., Cotton W. D., Greisen E. W., Yin Q. F., Perley R. A., Taylor G. B., Broderick J. J., 1998, AJ, 115, 1693

Crawford F. et al., 2013, ApJ, 776, 20

D'Amico N., Possenti A., Manchester R. N., Sarkissian J., Lyne A. G., Camilo F., 2001, ApJL, 561, L89

Demorest P. B., Pennucci T., Ransom S. M., Roberts M. S. E., Hessels J. W. T., 2010, Nature, 467, 1081

Eastman J., Siverd R., Gaudi B. S., 2010, PASP, 122, 935

Eggleton P. P., 1983, ApJ, 268, 368

Guillemot L. et al., 2012, MNRAS, 422, 1294

He C., Ng C.-Y., Kaspi V. M., 2013, ApJ, 768, 64

Hessels J. W. T., Ransom S. M., Stairs I. H., Freire P. C. C., Kaspi V. M., Camilo F., 2006, Science, 311, 1901

Hessels J. W. T. et al., 2011, in American Institute of Physics Conference Series, Vol. 1357, American Institute of Physics Conference Series, Burgay M., D'Amico N., Esposito P., Pellizzoni A., Possenti A., eds., pp. 40-43

Kaplan D. L., Bhalerao V. B., van Kerkwijk M. H., Koester D., Kulkarni S. R., Stovall K., 2013, ApJ, 765, 158

Kong A. K. H. et al., 2012, ApJL, 747, L3

Kurucz R. L., 1993, SYNTHE spectrum synthesis programs and line data

Li M., Halpern J. P., Thorstensen J. R., 2014, ApJ, accepted (arxiv 1409.3877) 
Linares M., 2014, ApJ, 795, 72

Marsh T. R., 1989, PASP, 101, 1032

Morris S. L., 1985, ApJ, 295, 143

Nolan P. L. et al., 2012, ApJS, 199, 31

Orosz J. A., Bailyn C. D., 1997, ApJ, 477, 876

Orosz J. A., van Kerkwijk M. H., 2003, A\&A, 397, 237

Pecaut M. J., Mamajek E. E., 2013, ApJS, 208, 9

Pletsch H. J. et al., 2012, ApJ, 744, 105

Predehl P., Schmitt J. H. M. M., 1995, A\&A, 293

Ray P. S. et al., 2012, 2011 Fermi Symposium proceedings - eConf C110509; ArXiv 1205.3089

Roberts M. S. E., 2011, in American Institute of Physics Conference Series (arXiv: 1103.0819), Vol. 1357, American Institute of Physics Conference Series, Burgay M., D'Amico N., Esposito P., Pellizzoni A., Possenti A., eds., pp. $127-130$

Roberts M. S. E., 2013, in IAU Symposium (arXiv:1210.6903), Vol. 291, IAU Symposium, pp. $127-132$

Romani R. W., 2012, ApJL, 754, L25

Romani R. W., Filippenko A. V., Silverman J. M., Cenko S. B., Greiner J., Rau A., Elliott J., Pletsch H. J., 2012, ApJL, 760, L36

Salvetti D. et al., 2015, ApJ, 814, 88

Saz Parkinson P. M., Xu H., Yu P. L. H., Salvetti D., Marelli M., Falcone A. D., 2016, ApJ, 820, 8

Schroeder J., Halpern J., 2014, ApJ, 793, 78

Shahbaz T., van der Hooft F., Charles P. A., Casares J., van Paradijs J., 1996, MNRAS, 282, L47

Skrutskie M. F. et al., 2006, AJ, 131, 1163

Smith J. A. et al., 2002, AJ, 123, 2121

Stellingwerf R. F., 1978, ApJ, 224, 953

Strader J., Chomiuk L., Sonbas E., Sokolovsky K., Sand D. J., Moskvitin A. S., Cheung C. C., 2014, ApJL, 788, L27

van Kerkwijk M. H., Breton R. P., Kulkarni S. R., 2011, ApJ, 728, 95

Wade R. A., Horne K., 1988, ApJ, 324, 411

Wright E. L. et al., 2010, AJ, 140, 1868

\section{APPENDIX A: IAC80 J021210.8+532032.5: A NEW W UMA CONTACT BINARY}

As a byproduct of our search, we report in this appendix the discovery and fundamental properties of a new W Ursae Majoris (W UMa) system, an eclipsing late-type contact binary system. We name this object 3 IAC80 J021210.8+532032.5 and locate it at R.A. $=02^{h} 12^{m} 10.77^{s}, \mathrm{DEC}=+53^{\circ} 20^{\prime} 32.5^{\prime \prime}$ (J2000), with an $0.4^{\prime \prime}$ error radius (FWHM/2). The light curve folded at the orbital period (Fig. A1) shows broad maxima around phase 0.25 and 0.75 , and narrower minima (flat in some cases, indicative of eclipses) around phase 0.5 and 1 . We do not detect colour variations along the orbit, and constrain any changes in g'-r' and r'-i' to be smaller than $\sim 0.05$ mag. These are all typical properties of W UMa-type binaries.

On 2015 February 27, we obtained seven 10-min

3 Not listed in the following online W UMa catalogues: http://astro.utoronto.ca/r̃ucinski/ogle.html http://www.oa-roma.inaf.it/maceroni/wumacat.html http://www.konkoly.hu/staff/csizmadia/wuma.html

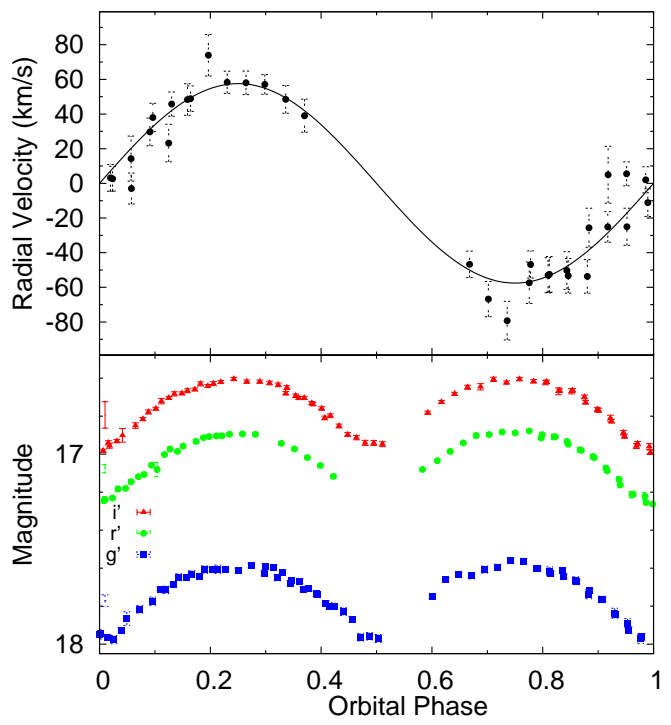

Figure A1. Top: Radial velocity curve of J0212-WUMa measured with WHT-ISIS in August 2015, folded at the orbital period $\left(\mathrm{P}_{\text {orb }}=7.469 \mathrm{~h}\right)$. The best-fit sine function is shown with a solid line (systemic velocities removed). Bottom: Phase-folded optical light curves of J0212-WUMa in three bands, as indicated, measured with IAC80-CAMELOT in February 2015. Error bars show the statistical error on the differential magnitude and are typically smaller than the symbols, while average calibration uncertainties are displayed along the left axis.

low-resolution ( $\left.\sim 500 \mathrm{~km} \mathrm{~s}^{-1}\right)$ WHT-ACAM-V400 spectra of IAC80 J021210.8+532032.5, covering the 4500-9400 A range. We reduced and extracted the spectra using standard procedures in IRAF and PAMELA, including subtraction of the bias level, flat-fielding and wavelength calibration with arc lamps fine-tuned with sky lines. The ACAM spectra of IAC80 J021210.8+532032.5 (taken during orbital phase 0.15-0.42) show hydrogen Balmer $(\mathrm{H} \alpha, \mathrm{H} \beta)$, sodium (NaI), calcium (CaII), iron (FeI) and magnesium (MgI) absorption lines, with little or no variability in intensity and width over the $1.2 \mathrm{~h}$ observation. These spectral features are typical of a G0 main sequence star, and we infer a F5-G5 spectral type using the same techniques described in the main text.

In order to measure the radial velocity curve, we obtained 33 medium-resolution WHT-ISIS spectra on 2015 August 26 and 27 with the R1200 gratings. The resulting spectra, extracted using the same procedures described in Section 2.2 have an average dispersion of $\sim 0.5 \AA /$ pixel and a resolution of $\sim 50 \mathrm{~km} \mathrm{~s}^{-1}$ for the blue arm (4600-5400 $\AA$; because the sharper metallic lines are present mostly in the blue arm, we focus our analysis on this wavelength range). Cross-correlation with a G0 template spectrum reveals the radial velocity curve of IAC80 J021210.8+532032.5, with an orbital pe$\operatorname{riod} \mathrm{P}_{\text {orb }}=0.311 \pm 0.001 \mathrm{~d}$ (twice the photometric period), a relatively low semi-amplitude $\left(\mathrm{K}_{2}=50.8 \pm 2.8 \mathrm{~km} \mathrm{~s}^{-1}\right)$, a negative systemic velocity $\left(\gamma=-55.5 \pm 1.7 \mathrm{~km} \mathrm{~s}^{-1}\right)$ and a barycentric epoch of zero phase (inferior conjunction) T0=57262.125 \pm 0.002 MJD. Fig. A1 shows both the light and radial velocity curves folded at the orbital period. 